\title{
BMJ Open Observational study of the development and evaluation of a fertility preservation patient decision aid for teenage and adult women diagnosed with cancer: the Cancer, Fertility and Me research protocol
}

\author{
G L Jones, ${ }^{1}$ J Hughes, ${ }^{1} \mathrm{~N}$ Mahmoodi, ${ }^{1}$ D Greenfield, ${ }^{2}$ G Brauten-Smith, ${ }^{3} \mathrm{~J}$ Skull, ${ }^{4}$ \\ J Gath, ${ }^{5}$ D Yeomanson, ${ }^{6}$ E Baskind, ${ }^{7} \mathrm{~J}$ A Snowden, ${ }^{8,9} \mathrm{R} \mathrm{M}$ Jacques, ${ }^{10}$ \\ G Velikova, ${ }^{11} \mathrm{~K}$ Collins, ${ }^{12} \mathrm{D}$ Stark, ${ }^{13} \mathrm{R}$ Phillips, ${ }^{14} \mathrm{~S}$ Lane, ${ }^{15} \mathrm{H}$ L Bekker, ${ }^{16}$ \\ (On behalf of the Cancer, Fertility and Me research team)
}

To cite: Jones GL, Hughes J, Mahmoodi N, et al. Observational study of the development and evaluation of a fertility preservation patient decision aid for teenage and adult women diagnosed with cancer: the Cancer, Fertility and Me research protocol. BMJ Open 2017;7:e013219.

doi:10.1136/bmjopen-2016013219

- Prepublication history for this paper is available online. To view these files please visit the journal online (http://dx.doi.org/10.1136/ bmjopen-2016-013219).

Received 28 June 2016 Revised 12 December 2016 Accepted 19 December 2016

CrossMark

For numbered affiliations see end of article.

Correspondence to

Professor GL Jones; g.l.

jones@leedsbeckett.ac.uk

\section{ABSTRACT}

Introduction: Women diagnosed with cancer and facing potentially sterilising cancer treatment have to make time-pressured decisions regarding fertility preservation with specialist fertility services while undergoing treatment of their cancer with oncology services. Oncologists identify a need for resources enabling them to support women's fertility preservation decisions more effectively; women report wanting more specialist information to make these decisions. The overall aim of the 'Cancer, Fertility and Me' study is to develop and evaluate a new evidence-based patient decision aid (PtDA) for women with any cancer considering fertility preservation to address this unmet need.

Methods and analysis: This is a prospective mixedmethod observational study including women of reproductive age ( 16 years + ) with a new diagnosis of any cancer across two regional cancer and fertility centres in Yorkshire, UK. The research involves three stages. In stage 1, the aim is to develop the PtDA using a systematic method of evidence synthesis and multidisciplinary expert review of current clinical practice and patient information. In stage 2, the aim is to assess the face validity of the PtDA. Feedback on its content and format will be ascertained using questionnaires and interviews with patients, user groups and key stakeholders. Finally, in stage 3 the acceptability of using this resource when integrated into usual cancer care pathways at the point of cancer diagnosis and treatment planning will be evaluated.

This will involve a quantitative and qualitative evaluation of the PtDA in clinical practice. Measures chosen include using count data of the PtDAs administered in clinics and accessed online, decisional and patient-reported outcome measures and qualitative feedback. Quantitative data will be analysed using descriptive statistics, paired sample t-tests and Cls; interviews will be analysed using thematic analysis.

\section{Strengths and limitations of this study}

- To the best of our knowledge, this research will develop the first, open access, evidence-based fertility preservation decision aid that is suitable for women of reproductive age (16 years+) and diagnosed with any cancer.

- The research will provide evidence of its acceptability and utility to women and healthcare professionals in usual practice across cancer and fertility care pathways.

- The research will provide evidence for the causal assumptions of its effectiveness and issues for implementation in usual care practice.

- This research will not provide evidence of its effectiveness on healthcare outcomes. However, our findings will provide the evidence to inform the study design for evaluating the effectiveness of this complex intervention on health outcomes in the future.

Ethics and dissemination: Research Ethics Committee approval (Ref: 16/EM/0122) and Health Research Authority approval (Ref: 194751) has been granted. Findings will be published in open access peer-reviewed journals, presented at conferences for academic and health professional audiences, with feedback to health professionals and program managers. The Cancer, Fertility and Me patient decision aid (PtDA) will be disseminated via a diverse range of open-access media, study and charity websites, professional organisations and academic sources. External endorsement will be sought from the International Patient Decision Aid Standards (IPDAS) Collaboration inventory of PtDAs and other relevant professional organisations, for example, the British Fertility Society.

Trial registration number: NCT02753296; pre-results. 


\section{INTRODUCTION}

\section{The impact of cancer treatment on female fertility}

Approximately $50 \%$ of people in the UK will be diagnosed with cancer at some point in their lifetime. Owing to rising survival rates, the importance of addressing the late effects of cancer treatment, such as the risk of infertility, has increased. ${ }^{2}$ Chemotherapy, radiotherapy, hormonal, medical and surgical interventions may all affect female fertility. Although, the degree to which chemotherapy and radiotherapy impacts on gonadal function depends on the treatment agent administered, the dose, as well as the woman's age and levels of ovarian reserve at the time, ${ }^{3-5}$ loss of fertility is considered one of the most significant late effects of cancer treatment ${ }^{3}$ and cancer survivors often report it as one of the most distressing outcomes of their cancer treatment. ${ }^{6}$

\section{The rationale for the study}

Women diagnosed with cancer have to make timepressured decisions about fertility preservation while simultaneously planning their cancer treatment. These decisions are stressful as women are having to trade-off the immediate consequences of starting cancer treatment with the long-term chances of having a biological child in the future, post cancer treatment. For those contemplating fertility preservation, women are required to move between two medical services (oncology and fertility services) to make care planning decisions collaboratively with fertility preservation and cancer teams.

In the initial stages of treatment for cancer, the American Society of Clinical Oncology and the American Society for Reproductive Medicine recommend options to preserve fertility which are discussed with each patient of reproductive age about to undergo cancer treatment which may affect their fertility. ${ }^{78}$ The current National Institute for Clinical Excellence (NICE) pathway for preserving fertility in people diagnosed with cancer presents similar guidelines for the UK. ${ }^{9}$ However, the evidence suggests that many women do not feel well supported in their choices, with many missing out on having fertility preservation at this crucial time. A recent UK survey by Breast Cancer Care found that as little as $12 \%$ of 170 women were referred to a fertility consultant, with many being unaware that infertility was a consequence following chemotherapy. ${ }^{10}$

Evidence from the medical and psychological literature examining aspects of fertility, pregnancy and decision-making following a cancer diagnosis have identified a range of factors which may hinder decisionmaking for this patient population. ${ }^{11-25}$ The barriers identified are diverse including financial concerns (especially in those countries where fertility preservation is not covered by insurance), fear associated with aggravating a hormone-sensitive cancer or a future pregnancy (in terms of a cancer recurrence and/or implications for the health of a future child) and lack of referral to fertility services (eg, due to reasons such as the oncologist prioritising cancer survival).

However, lack of fertility preservation information is also a key reason cited. The need for more evidencebased information that is integrated into the cancer care pathway early, and prior to transition to fertility services, has been identified as an important factor to facilitate women's decision-making at this time. ${ }^{26}{ }^{27}$ It has been found that oncologists lack specific fertility preservation information for patients, and have only moderate confidence in their knowledge about fertility and the preservation options available. ${ }^{28}$ Therefore, they have also expressed the need for more evidence-based fertility preservation information to enable them to support women's decision-making more effectively. ${ }^{29}$

\section{Patient decision aids}

Patient decision aids (PtDAs) are information resources supporting people to make decisions between healthcare options. ${ }^{30}$ They are evidence-based resources, drawing on clinical effectiveness of healthcare options data, studies of patients' decision-making and illness experiences and evidence from the decision sciences on how people make healthcare choices. ${ }^{31}$ PtDAs support people to make reasoned decisions, that is, ones based on accurate information about the consequences of all options, in accordance with their beliefs, and trade-offs between their treatment preferences. ${ }^{32-34}$ Compared with usual care, receiving a PtDA helps people participate with their health professionals in making personalised choices between healthcare options. ${ }^{32} 33$ They have been shown to improve patients' knowledge of the risks and benefits of options, value of consequences to their lives and efficacy in making informed decisions. ${ }^{30}$

While there are many fertility preservation resources publically available for women with cancer, ${ }^{35}$ few exist to support the fertility preservation decision process in women of reproductive age. ${ }^{35}$ Of those publishing their development studies, two PtDAs were designed for women with breast cancer specifically, and none for women in the UK, ${ }^{36}{ }^{37}$ despite women with a range of different cancers facing fertility preservation treatment decisions. It is therefore likely a new PtDA supporting women diagnosed with any cancer to make fertility preservations decisions will meet patient, service and practice needs. ${ }^{25} 2635$

\section{AIMS AND OBJECTIVES}

The aim of this research is to develop a fertility preservation PtDA enabling cancer and fertility services to support effectively women's fertility preservation decisions following a diagnosis of any cancer type.

Our objectives are to:

1. develop a PtDA for use by oncology and haematology teams to support women making fertility preservation choices, while having a recent cancer diagnosis; 
2. assess the face validity of the PtDA to support women making informed decisions about fertility preservation before starting their cancer treatment and

3. evaluate the acceptability of the PtDA using qualitative and quantitative methods to 1 ) women making fertility preservation decisions while planning their cancer treatment and 2) oncology, haematology and fertility health professionals supporting women's cancer and fertility treatment choices.

Evaluation of the PtDA should enable us to determine whether the provision of a PtDA early in the cancer care pathway better supports women, especially in the stressful intervening period between planning their cancer treatment and referral to the fertility expert. We also anticipate that the provision of this PtDA will enable women to make more informed fertility preservation treatment decisions, have more focused consultations with the fertility experts and a better opportunity to ask the right questions for them at the right time.

\section{METHODS AND ANALYSIS}

All aspects of the research are discussed with the steering group, and all stakeholders comment on all the materials. There has been patient and public involvement in the development of the research protocol and initial needs assessment. The PtDA is being developed across two regional cancer (adult, and teenage and young adult services) and fertility centres in Yorkshire. Ethics approval was granted on 5 April 2016.

\section{Design}

The Cancer, Fertility and Me PtDA was developed over a year using systematic and evidence-based methods (table 1-stages 1 and 2). ${ }^{33} 3839$ A prospective, observational study using interview and questionnaire methods to evaluate the PtDA will be conducted (table 1-stage 3) informed by Medical Research Council Guidance for Developing Complex Interventions. ${ }^{40}$

\section{Stage 1: Development of the PtDA (November 2015-July 2016)}

Stage 1 has used evidence to develop a PtDA supporting women to make informed decisions about fertility preservation options before starting cancer treatment. This will be for use alongside usual cancer and fertility care pathways.

Table 1 The three stages of the Cancer, Fertility and Me study

\begin{tabular}{|c|c|c|c|}
\hline Stages & Methods & Data collection & Sample \\
\hline $\begin{array}{l}\text { Stage 1: } \\
\text { development of } \\
\text { the PtDA }\end{array}$ & $\begin{array}{l}\text { Identifying the } \\
\text { active ingredients } \\
\text { of the PtDA }\end{array}$ & $\begin{array}{l}\text { IPDAS checklist and evidence articles } \\
\text { used as a framework for development. } \\
\text { Evidence synthesis of women with } \\
\text { cancer's experience of making FP } \\
\text { decisions. Evidence from clinical } \\
\text { guidelines and best practice. } \\
\text { Review of current practice-service } \\
\text { delivery and patient resources. } \\
\text { Iterative development process. }\end{array}$ & $\begin{array}{l}\text { Study team, steering group with } \\
\text { cross-sector expertise, oncologists, } \\
\text { haematologists, fertility experts, decision } \\
\text { scientist, relevant charity organisations, } \\
\text { stakeholders and service users/PPI } \\
\text { panel supporting the study. }\end{array}$ \\
\hline \multirow[t]{2}{*}{$\begin{array}{l}\text { Stage } 2 \text { : face } \\
\text { validity }\end{array}$} & Quantitative & $\begin{array}{l}\text { LV questionnaire (comprising of } 4 \text { items } \\
\text { taken from the QQ-10 and some open } \\
\text { questions), and the Preparation for } \\
\text { Decision-making questionnaire. }\end{array}$ & $\begin{array}{l}10 \text { women ( } 5 \text { from each site). } \\
10 \text { health professionals ( } 5 \text { from each } \\
\text { site). } \\
+ \\
\text { Women and key health professionals } \\
\text { from the relevant user groups and } \\
\text { organisations identified by The Cancer, } \\
\text { Fertility and Me steering group and } \\
\text { systematic reviews. }\end{array}$ \\
\hline & Qualitative & Semi-structured telephone interviews. & $\begin{array}{l}\text { The same } 10 \text { women and health } \\
\text { professionals. } \\
+ \\
\text { Additional women and key health } \\
\text { professionals described above. }\end{array}$ \\
\hline $\begin{array}{l}\text { Stage 3: } \\
\text { evaluation } \\
\text { 3a }\end{array}$ & $\begin{array}{l}\text { Quantitative } \\
\text { (baseline, time 1, } \\
\text { time 2a) }\end{array}$ & $\begin{array}{l}\text { EQ-5D, State Trait Anxiety. } \\
\text { Inventory, Stage Of Decision-Making, } \\
\text { Decisional Conflict Scale, preparation for } \\
\text { decision-making, count data. }\end{array}$ & 78 women (in total from both sites). \\
\hline $3 b$ & $\begin{array}{l}\text { Qualitative (time } \\
2 \mathrm{~b} \text { ) }\end{array}$ & $\begin{array}{l}\text { Semi-structured interviews, EQ-5D, } \\
\text { Decisional Regret Scale. }\end{array}$ & $\begin{array}{l}30 \text { women and health professionals (in } \\
\text { total from both sites). }\end{array}$ \\
\hline
\end{tabular}

EQ-5D, EuroQol five dimensions questionnaire; FP, fertility preservation; IPDAS, International Patient Decision Aid Standards; PPI, Patient and Public Involvement; PtDA, patient decision aid. 
The PtDA has used guidance from the International Patient Decision Aid Standards (IPDAS) collaboration ${ }^{34}$ on balance of options, ${ }^{41}{ }^{42}$ risk presentation, ${ }^{43-45}$ eliciting values, ${ }^{46}{ }^{47}$ use of patient stories, ${ }^{30}$ enabling readability ${ }^{48}$ and understanding illness. ${ }^{49} 50$ The guidance helps information to be structured so that it encourages people to evaluate all decision options, and their consequences, in accordance with their values and without bias, thus, enabling decision-making to be based on their trade-offs between these evaluations, that is, to make a reasoned decision. ${ }^{34}$

The aim is for women to receive the PtDA from their cancer health care professionals as part of usual care during the patient's first consultation to discuss cancer and/or fertility treatment options (this could be at cancer diagnosis), before referral to fertility services. The PtDA is being disseminated as a leaflet and PDF on a website, and evaluated accordingly. The content of the PtDA will be informed by evidence from the following:

- Clinical guidelines on infertility and cancer prognosis, risks and benefits of cancer and fertility preservation treatment. ${ }^{8-9} 35$

- Systematic narrative review of women's values, treatment preferences and decision-making experiences about fertility preservation when diagnosed with cancer, completed in June 2016. This was carried out as part of the previous PreFer study which was a 3-year mixed-methods prospective study exploring fertility preservation decision-making and quality of life in women with cancer. ${ }^{25}$

- Environmental scan using systematic methods to synthesise evidence about open access resources for women with cancer about fertility preservation (patient information and clinical guidelines) and critically evaluate their ability to support informed decision-making, completed in May 2016. ${ }^{35}$

- Observations of local service delivery, referral pathways within and across services and integration of research practices across regional National Health Service (NHS) centres for cancer and infertility services, completed by June 2016.

Regular meetings with the study steering group to decide the content and design of the PtDA. During this time web and graphic designers were involved in developing the PtDA's identity for use in health and patient forums, completed by July 2016.

\section{Stage 2: Face validity study (July 2016-December 2016)}

Stage 2 assesses the face validity of the PtDA for stakeholders. During the development of decision aids, this process is sometimes referred to as Learner Verification (LV). ${ }^{51}$ The aim is to assess the PtDA across stakeholders for attractiveness, comprehension, cultural acceptability, self-efficacy and persuasion. ${ }^{52} 53$

\section{Sample}

A purposive sample of eligible women and health professionals will be invited to participate from the study sites.
All women of reproductive age (16 years + ), diagnosed with any cancer and undergoing or has undergone cancer treatment(s) which may impact fertility will be eligible. The sample of health professionals will consist of adult and paediatric oncologists and haematologists, cancer surgeons, cancer nurse specialists and fertility specialists (clinicians, nurses and counsellors).

There is no statistical guidance for undertaking LV methods using qualitative methods. Prior literature and our research experience suggest a sample size of 20 participants in total across both sites to be appropriate. ${ }^{54}$ Therefore, 10 women and 10 health professionals from the two clinical centres will be invited to participate. In addition to the 20 participants, women and key health professionals will be invited to participate from relevant user groups/forums and professional organisations identified by the Cancer, Fertility and Me steering group and our systematic reviews (eg, National Cancer Research Initiative, Breast Cancer Care and British Fertility Society among others).

\section{Recruitment}

To recruit the women to stage 2, the nurses/clinicians will make the initial approach (for those women recruited from the two clinical centres). For the women recruited through the service user groups/forums, the lead contacts for the service user groups will make the initial approach. Following this, the contact details of the interested women will be passed to a trained researcher and those willing to participate will be sent the PtDA and associated documents to review by post. Recruitment of health professionals will be obtained from the study sites and through purposive and snowball sampling for the key stakeholders. The PtDA will be sent by post or via a PDF online depending on the request of the health professional. Appropriate consenting procedures and guidelines prescribed by the British Psychological Society ${ }^{55}$ and NHS research protocols will be followed.

\section{Data collection}

All the women and health professionals will be asked to complete a study questionnaire which includes the LV questionnaire, and the Preparation for Decision-making questionnaire. ${ }^{56}$ The LV questionnaire will consist of four items taken from the QQ- $10,{ }^{57}$ but will also comprise of three open-ended questions relating to the acceptability and utility of the PtDA from women's and health professional's perspective. The Preparation for Decision-making scale ${ }^{56}$ is a 10 -item measure which assesses an individual's perception of how useful a PtDA is in preparing the respondent to communicate with their practitioner at a consultation focused on making a health decision. High scores on the overall scale (range 0-100) indicate higher perceived levels of preparation for decision-making.

Second, all the health professionals and women who completed the questionnaire and consent form, will be asked to take part in a follow-up telephone interview. An 
interview schedule will be used to seek their feedback and understanding of the purpose of the PtDA and study measures. ${ }^{51}{ }^{53}$ Interviews will be audio recorded, digitalised and transcribed for analysis.

\section{Data analysis}

Telephone interviews will be coded and managed using NVivo 10 qualitative data analysis software. Analysis will use a practical, thematic approach outlined by Braun and Clarke ${ }^{58}$ using a systematic five-step approach: familiarisation, generating initial codes, searching for themes, reviewing themes and defining and naming themes.

\section{Stage 3: Evaluation study ( January 2017-May 2018)}

Stage 3 evaluates the acceptability, feasibility and usefulness of the PtDA in clinical practice with women and health professionals. The study design employs mixed methods, using quantitative (stage $3 \mathrm{a}$ ) and qualitative methods (stage $3 \mathrm{~b}$ ).

\section{Stage 3a Quantitative sample}

All women receiving a new diagnosis of cancer from two regional centres will be eligible for participation. Inclusion criteria are women of a reproductive age $(16$ years +$)$ with a new diagnosis of any cancer, and facing cancer treatment(s) with curative intent, which may impact fertility. We anticipate that the majority will be women with breast cancer, although women with lymphoma and leukaemia, head and neck cancers and cervical cancers are also likely to be represented in this research. From the results of our previous PreFer study, ${ }^{13}$ we have found that $\sim 90$ women, across both hospital sites, aged between 16 and 40 years are diagnosed with cancer and face chemotherapy treatment annually and are eligible to receive the new PtDA. We identified around $17 \%$ of women from this group do not wish to consider fertility preservation as they already have children or never wanted children. During the 12-month period we hope to recruit $\sim 78$ women in total, taking into account a $20 \%$ non-response rate from our previous data. Using a paired t-test to compare the outcome measures before and after the implementation of the PtDA, with 78 participants, we have $80 \%$ power at $5 \%$ two-sided significance to detect a minimum standardised effect size of 0.32 .

\section{Stage 3a Quantitative recruitment}

We will adopt the 'referral model' for implementing the PtDA $^{59}$ that is, the PtDA will be mentioned and discussed by their healthcare professional with any woman fitting the eligibility criteria during the patient's first consultation to discuss treatment options. The referral model proposes that these tools are 'adjuncts' that support decision-making, when used ahead of visits, or shortly afterwards. ${ }^{59}$ Eligible women will be invited to participate by the researchers working with the clinical care team, immediately following this consultation.
Stage 3a Quantitative data collection

Quantitative data will be collected at three time-points (figure 1). Questionnaire packs will be given to women at the same time as the PtDA (baseline); the timing of their attendance at the fertility clinic or cancer treatment starting point if not going to fertility services (time 1) and after their first session of chemotherapy (time 2a). The measures chosen for this study have been decided on following recommended guidance from the Patient Decision Aid International Research Group, ${ }^{60}$ and will assess the use of the PtDA and decisional preparedness.

Baseline: questionnaire pack includes: The State Trait Anxiety Inventory-STAI-6, ${ }^{61}$ the EuroQol five dimensions questionnaire (EQ-5D) ${ }^{62}$ the Stage of Decision-making ${ }^{63}$ and the Decisional Conflict Scale. ${ }^{64}$ Women are instructed not to open and read the PtDA (or access it online) until they have completed the questionnaires.

The State Trait Anxiety Inventory-6 item ${ }^{45}$ is a brief 6 -item version used to measure of state anxiety. All items are rated on a 4-point scale (1-Almost never, 4-Almost always). Higher scores indicate greater anxiety. The EQ- $5 \mathrm{D}^{62}$ is a standardised instrument for use as a measure of health outcome. It consists of five dimensions: mobility, self-care, usual activities, pain/discomfort and anxiety/depression. Each dimension has three levels: no problems, some problems and extreme problems. The Stage of Decision-making ${ }^{63}$ is a 6-category tool to assess the individual's readiness to engage in decision-making, progress in making a choice and receptivity to considering or reconsidering options. The tool is rated on a 6-point scale (1-not thinking about it at all; 6-considered the options). The Decisional Conflict Scale $^{64}$ is a 16 -item scale which measures the patient's reported experience of making a reasoned and/or conflicted decision.

Scores $>37.5$ on the overall scale (range 0-100) indicate high decisional conflict, which is characterised by decision delay and/or uncertainty about decision.

Time 1: Questionnaire pack includes: the STAI- $6{ }^{45}$ the Stage of Decision-making ${ }^{63}$ and the Preparation for Decision-making. ${ }^{56}$

Time 2a: Questionnaire pack includes: the STAI-6, ${ }^{45}$ the Stage of Decision-making ${ }^{63}$ and the Decisional Conflict Scale. ${ }^{64}$

\section{Stage 3a Quantitative data analysis}

We will report summary statistics for the count data and other service indicators. For the decisional outcome measures and other patient-reported outcomes we will use paired sample t-tests to calculate mean change in scores from baseline to time 1 and from baseline to time 2. $95 \%$ CIs for the mean changes will also be calculated. All statistical tests will be two-tailed with significance determined at $\mathrm{p} \leq 0.05$. 


\section{Stage 3b Qualitative sample}

Qualitative, semistructured interviews with a purposive sample of $\sim 30$ women from the stage 3 a evaluation (15 women from each site) and 30 health professionals including cancer surgeons, adult and paediatric oncologists and haematologists, nurse specialists and fertility experts (15 from each site) will be carried out. The purpose of the interviews are to gain a deeper sight into service user and healthcare professionals experiences of using the PtDA and its impact on the decision- making process and clinical care, within the context of this complex intervention. ${ }^{40}$ Although a total of $\sim 30$ interviews are estimated, this will be guided by data saturation, following established protocols in qualitative research. ${ }^{586566}$

\section{Stage 3b Qualitative recruitment}

The sample will consist of the women recruited into the stage 3 quantitative study, and the clinical sample will be obtained through snowball sampling methods. Women and health professionals will be issued with a study pack, including a study information sheet and the PtDA. On the day of the interview, an interview consent form will be completed. As shown in

Figure 1, the interview with women and the health professionals will be carried out after the first round of chemotherapy has been completed (time 2b) at a place that is most preferred.

\section{Stage 3b Qualitative data collection}

The interview schedule will comprise of the telephone interview LV schedule used in stage 2, with additional open-ended questions to add depth and breadth to the interpretation of the quantitative results enabling further insight into their experiences. It will also focus on LV and the PtDAs clarity and usefulness in planning care and making decisions between treatment options. However, additional areas we will explore include the PtDAs likelihood of use, barriers to use in practice, whether or not the women and health professionals benefit from their delivery, usefulness of the PtDA in aiding service transition and how the women used the PtDA.

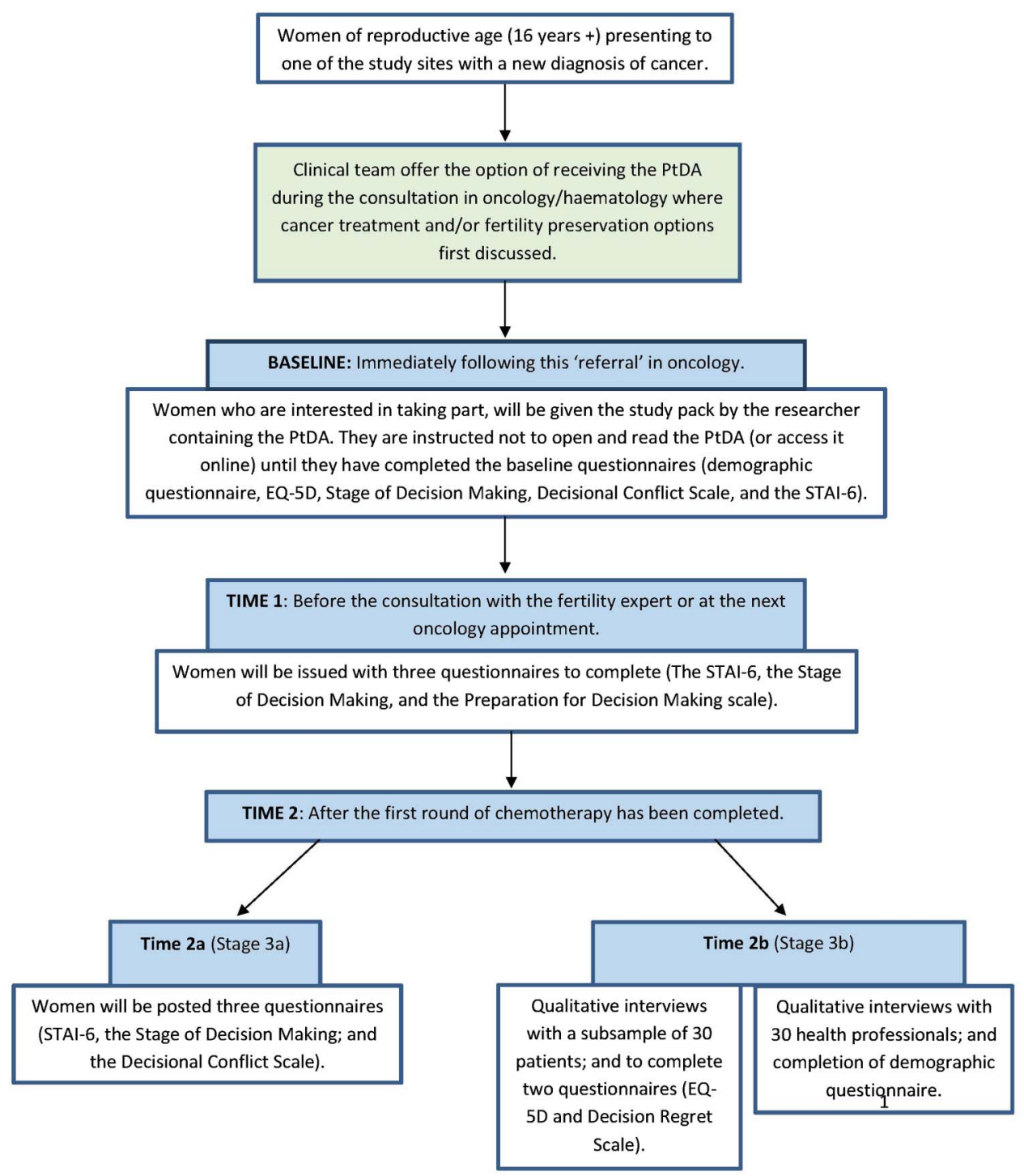


All interviews will be audio-recorded and transcribed verbatim for analysis. Following the interview, the women will be given a final study pack questionnaire to complete, which comprises of the EQ-5D ${ }^{62}$ and the Decisional Regret Scale. ${ }^{67}$ The Decisional Regret Scale is a brief five-item scale which measures 'distress or remorse after a health care decision' using a 5-point Likert scale (1- strongly agree; 5- strongly disagree). A score of 0 on the overall scale (range $0-100$ ) indicates no regret; scores of 100 mean high regret.

\section{Stage 3b Qualitative data analysis}

Interviews will be coded and managed using NVivo 10 . Framework analysis will be carried out to identify recurrent themes which have been specifically developed for applied health and policy research. ${ }^{68}$ Independent analysis of the transcribed data by members of the study team and steering group will take place. Interdisciplinary analysis meetings will include critical appraisal of the literature, systematic data and coding framework verification and challenging of interpretive analysis. We will map the data against the themes identified from the existing literature as well as allow new themes to emerge. We will adhere to established quality criteria for qualitative research. ${ }^{586566}$

\section{Other questionnaires and outcome measures}

Demographic information about women's age, ethnicity, employment and treatment status will be collected through a questionnaire during stage 2 (face validity) and at stage 3 baseline. Demographic information about health professionals will also be collected through a questionnaire, during stage 2 and at stage 3 (time $2 \mathrm{~b}$ ). Details of patients' cancer treatment(s) will be recorded at the end of the study using a study 'cancer treatment proforma'. This proforma has been ethically approved and used to record the cancer treatment details in the previously mentioned PreFer Study. ${ }^{26}$

Count data will be collected of the number of PtDAs given to women and health professionals, counts of use and number of clicks on the 'Cancer, Fertility and Me' website and downloads of the online PDF version will be recorded. In addition, we will record length of oncology, haematology and fertility consultations and length of time to fertility and cancer treatment. Using count data is a common method for evaluating a decision aid. ${ }^{69}$

\section{ETHICS AND DISSEMINATION \\ Ethical considerations}

Written, signed consent will be obtained from all participants. Issues particularly pertinent to this study will include participants' right to withdraw from the research process, informed consent and their right to confidentiality and anonymity. Usual NHS care will be provided by the health professionals involved, which includes referral to support and counselling services available within the oncology, haematology and fertility services at the NHS study sites, if any of the women wish. In line with good practice, across both sites, the young women aged 1617 years and their parents/guardians will be asked to sign for consent.

\section{Dissemination}

This research will involve rigorous methods and evaluation in a clinical context. ${ }^{40}$ The multidisciplinary and collaborative nature of this proposal will enable us to disseminate the research and its milestones to the study participants and into the NHS and wider healthcare community through a variety of local, national and international channels regularly throughout the duration of this research.

During the development and evaluation phases of the research, we are engaging with patients and the public through the North Trent Consumer Research Panel group, NHS England's Patient Involvement Team, International Cancer Patient Voices, the service user

research partnership of Breast Cancer Care as well as presenting our findings at INVOLVE national conferences. A study website is currently under development (http://www.leedsbeckett.ac.uk/cancerfertilityandme.ac. uk).

It will be used to provide an evidence-based portal on the study, team and links with our international partners. It will enable access to the web-based version of the instrument and provide two-way links into other relevant organisations, charities signposted on our webpage and the other social media planned to be used in the study, for example, twitter/blogs. We plan to impact the academic and clinical community more widely through conference presentations and publications in peer-reviewed journals that are open access.

Findings on the quality of the PtDA and study outcomes will be used to provide feedback to health professionals and programme managers. Audit and feedback is an effective intervention for changing the behaviour of health professionals (Cochrane Effective Practice and Organisation of Care Group). The study will be widely and regularly disseminated throughout its duration.

We will develop links with NHS England patient information teams and other UK and international bodies who endorse patient education materials for use/dissemination by service teams. We will also seek assessment by the international criteria of PtDAs to be included in their A to $\mathrm{Z}$ inventory. ${ }^{60}$

The primary output of this study will be the final version of the PtDA. Once evaluated, it will be promoted widely and made available free through a diverse range of media (ie, social and print), charity websites, professional organisations, academic sources and posted to all key stakeholders and participants.

\section{DISCUSSION}

It is anticipated that this research will provide evidence about the effectiveness of a PtDA to support cancer 
patients' decision-making in relation to fertility preservation. Currently, fertility preservation PtDAs for women of reproductive age only exist to support women with breast cancer. A unique feature of this research is that we hope to provide evidence that one open-access PtDA can be used effectively across a range of women's cancer types. Another strength of this research is that it will be administered early into the cancer care pathway, thus providing clinical cancer care teams with an evidencebased resource to provide to all women diagnosed with cancer, therefore meeting a current unmet need. It is anticipated that this will encourage more cancer specialists to have fertility related discussions with women, help raise fertility awareness and provide a resource that will improve the care, support and management of the women.

While this might result in more women choosing to see the fertility expert (Breast Cancer Care alone estimated that around 5000 women per year who should be having this consultation are missing out), it should also reduce the number of women with cancer who are inappropriately referred for fertility preservation and unsuitable for this treatment.

In trying to create a resource that is suitable for women with any cancer, this undoubtedly creates a number of challenges (in terms of synthesising the evidence on cancer treatment on female reproduction and the fertility preservation choices available). We have tried to prepare for this and included an extensive face validity stage during which the PtDA will undergo review from a large number of patients, service users and key stakeholders from a variety of oncology and fertility specialities.

The overall aim of the 'Cancer, Fertility and Me' study is to develop and evaluate a new evidence-based PtDA for women with any cancer considering fertility preservation. The data generated as part of this study should help us identify factors associated with its implementation in practice, and/or integration in care. During its evaluation, the cancer care clinical teams will hand out information about the study and the PtDA. We will capture data on the acceptability of this method of integration within care from the clinical teams. It may be our clinical teams think a short skills training session on using our PtDA within cancer and fertility services will support its implementation in usual practice. It is likely future research evaluating our PtDA's impact on health outcomes may therefore also need to include an assessment of shared decision-making training within an implementation study's process evaluation.

\footnotetext{
Author affiliations

${ }^{1}$ Department of Psychology, School of Social Sciences, Leeds Beckett University, City Centre Campus, Leeds, UK

${ }^{2}$ Department of Oncology, Sheffield Teaching NHS Hospitals Foundation Trust, Sheffield University, Sheffield, UK

${ }^{3}$ Breast Cancer Care, London, UK

${ }^{4}$ Jessop Wing, Sheffield Teaching Hospitals NHS Foundation Trust, Sheffield, UK

${ }^{5}$ Independent Cancer Patients' Voice, London, UK
}

${ }^{6}$ Sheffield Children's Hospital NHS Foundation Trust, Sheffield, UK

${ }^{7}$ Seacroft Hospital, Leeds Teaching Hospitals, Leeds, UK

${ }^{8}$ Department of Haematology, Sheffield Teaching Hospitals NHS Foundation

Trust, Royal Hallamshire Hospital, Sheffield

${ }^{9}$ Department of Oncology and Metabolism, University of Sheffield, Sheffield, UK

${ }^{10}$ School of Health and Related Research, University of Sheffield, Sheffield, UK

${ }^{11}$ University of Leeds, St James Hospital, Leeds Teaching Hospitals, Leeds, UK

${ }^{12}$ Centre for Health and Social Care Research, Sheffield Hallam University,

Sheffield, UK

${ }^{13}$ University of Leeds, St James Hospital, Leeds Teaching Hospitals, Leeds, UK

${ }^{14}$ Center for Review and Dissemination, University of York, Leeds General

Infirmary, Leeds Teaching Hospitals, York, UK

${ }^{15}$ Oxford Radcliffe Hospitals NHS Trust, Oxford, UK

${ }^{16}$ Leeds Institute of Health Sciences, School of Medicine, University of Leeds, Leeds, UK

Contributors GLJ conceived the idea, secured funding and is the chief investigator. The first REC approval was made by GLJ. GLJ, JH, HLB, KC, DG, JAS, GV, GB-S, RMJ, JG provided intellectual input into the protocol for the grant application. GLJ, JH, HLB, NM, KC, DG, GV, GB-S, RMJ, JAS, EB, DY, $\mathrm{SL}, \mathrm{DS}, \mathrm{JG}, \mathrm{SL}, \mathrm{RP}$ provided intellectual input and study design for the final protocol of the study.

Funding This work is funded by Yorkshire Cancer Research, grant number (S391).

\section{Competing interests None declared.}

Ethics approval The study protocol was approved by the East Midlands NHS Research Ethics for the Protection of Persons of Bordeaux University (approval number 2015-A00778-41). It was also approved by the National Commission for Data Processing and Freedoms (approval number 1838811).

Provenance and peer review Not commissioned; externally peer reviewed.

Open Access This is an Open Access article distributed in accordance with the Creative Commons Attribution Non Commercial (CC BY-NC 4.0) license, which permits others to distribute, remix, adapt, build upon this work noncommercially, and license their derivative works on different terms, provided the original work is properly cited and the use is non-commercial. See: http:// creativecommons.org/licenses/by-nc/4.0/

\section{REFERENCES}

1. Sasieni PD, Shelton J, Ormiston-Smith $\mathrm{N}$, et al. What is the lifetime risk of developing cancer?: the effect of adjusting for multiple primaries. Br J Cancer 2011;105:460-5

2. Cancer Research UK. Cancer Stats for the UK (retrieved 26 Jan 2016). http://www.cancerresearchuk.org/health-professional/ cancer-statistics\#heading-Zero

3. Meirow D, Nugent $D$. The effects of radiotherapy and chemotherapy on female reproduction. Hum Reprod Update 2001;7:535-43.

4. Meirow D, Biederman $\mathrm{H}$, Anderson RA, et al. Toxicity of chemotherapy and radiation on female reproduction. Clin Obstet Gynecol 2010;53:727-39.

5. Maltaris T, Seufert R, Fischl F, et al. The effect of cancer treatment on female fertility and strategies for preserving fertility. Eur J Obstet Gynecol Reprod Biol 2007;130:148-55.

6. Peate $\mathrm{M}$, Meiser $\mathrm{B}$, Hickey $\mathrm{M}$, et al. The fertility-related concerns needs and preferences of younger women with breast cancer: a systematic review. Breast Cancer Res Treat 2009;116:215-23.

7. Lee SJ, Schover LR, Partridge AH, et al. American Society of Clinical Oncology recommendations on fertility preservation in cancer patients. J Clin Oncol Res 2006;24:2917-31.

8. The Ethics Committee of the American Society for Reproductive Medicine. Fertility preservation and reproduction in patients facing gonadotoxic therapies: a committee opinion Fertil Steril 2013;100:1224-3.

9. National Institute for Health and Care Excellence. Cryopreservation to preserve fertility in people diagnosed with cancer. http://pathways.nice. org.uk/pathways/fertility\#path=view\%3A/pathways/fertility/ cryopreservation-to-preserve-fertility-in-people-diagnosed-with-cancer. xml\&content=view-index (accessed 26 Jan 2016).

10. Breast cancer care study 'Standards of Care for Younger Women; Results from the survey of healthcare professionals' by Grete Brauten- Smith and Jennifer Finnegan-John. https://www. breastcancercare.org.uk/about-us/media/press-releases/ 
approximately-5000-breast-cancer-patients-missing-out-fertility-care (accessed 26 Jan 2016).

11. Dow KH, Kuhn D. Fertility options in young breast cancer survivors: a review of the literature. Oncol Nurs Forum 2004:31:E46-53.

12. Goossens J, Delbaere I, Van Lancker A, et al. Cancer patients' and professional caregivers' needs, preferences and factors associated with receiving and providing fertility-related information: a mixed-methods systematic review. Int J Nurs Stud 2014;51: 300-19.

13. Gonçalves V, Sehovic I, Quinn G. Childbearing attitudes and decisions of young breast cancer survivors: a systematic review. Hum Reprod Update 2014;20:279-92.

14. Holton BA, Kirkman M, Rowe $\mathrm{H}$, et al. The childbearing concerns and related information needs and preferences of women of reproductive age with a chronic, noncommunicable health condition: a systematic review. Womens Health Issues 2014;22:e541-52.

15. Howard-Anderson J, Ganz PA, Bower JE, et al. Quality of life, fertility concerns, and behavioural health outcomes in younger breast cancer survivors: a systematic review. J Natl Cancer Inst Monogr 2012;104:386-405

16. Jensen JR, Morbeck DE, Coddington CC. Fertility preservation. Mayo Clinical Procedure 2011;86:45-9.

17. Loren AW, Mangu PB, Beck LN, et al. Fertility preservation for patients with cancer: American Society of Clinical Oncology Clinical Practice Guideline Update. J Clin Oncol 2013;31:2500-10.

18. Quinn GP, Murphy D, Knapp C, et al. Who decides? Decision making and fertility preservation in teens with cancer: a review of the literature. J Adolesc Health 2011;49:337-46.

19. Schmidt R, Richter D, Sender A, et al. Motivations for having children after cancer--a systematic review of the literature. Eur J Cancer Care (Engl) 2016;25:6-17.

20. Sobota A, Ozakinci G. Fertility and parenthood issues in young female cancer patients-a systematic review. J Cancer Surviv 2014:8:707-21.

21. Penrose R, Beatty L, Mattiske J, et al. The psychosocial impact of cancer-related infertility on women. Clin J Oncol Nurs 2012:17:188-93.

22. Tschudin S, Bitzer J. Psychological aspects of fertility preservation in men and women affected by cancer and other life-threatening diseases. Hum Reprod Update 2009;15:587-97.

23. Zdenkowski N, Butow $\mathrm{P}$, Tesson $\mathrm{S}$, et al. Systematic review of decision aids for patients making a decision about treatment for early breast cancer. Breast 2016;26:31-45.

24. Lee MC, Gray J, Han HS, et al. Fertility and reproductive considerations in female premenopausal patients with breast cancer. Cancer Control 2010;17:162-72.

25. Jones GL, Hughes J, Mahmoodi N, et al. What factors influence the decision-making process for women with cancer contemplating fertility preservation? A systematic review. RCOG World Congress: Birmingham, UK. Abstract, July 2016.

26. Jones GL, Hughes J, Greenfield D, et al. What factors influence the fertility preservation treatment decision-making process in women with cancer? the qualitative findings of the PreFer study. European Shared Medical Decision Making conferences, London, UK: Abstract, July 2016

27. Wilkes $\mathrm{S}$, Coulson $\mathrm{S}$, Crosland $\mathrm{A}$, et al. Experience of fertility preservation among younger people diagnosed with cancer. Hum Fertil (Camb) 2010;13:151-8.

28. Duffy C, Allen SM, Dube C, et al. Oncologists' confidence in knowledge of fertility issues for young women with cancer. $J$ Cancer Educ 2012;27:369-76.

29. Adams E, Hill E, Watson E. Fertility Preservation in Cancer Survivors: a national survey of oncologists' current knowledge, practice and attitudes. Br J Cancer 2013;108:1602-15.

30. Stacey D, Bennett CL, Barry MJ, et al. Decision aids for people facing health treatment or screening decisions. Cochrane Database Syst Rev 2011;5:CD001431.

31. Bekker HL, Winterbottom AE, Butow P, et al. Do personal stories make patient decision aids more effective: a critical review of evidence and theory? BMC Med Inform Decis Mak 2013;13(Suppl 2):S9.

32. Rothert ML, Holmes-Rovner M, Rovner D, et al. An educational intervention as decision support for menopausal women. Res Nurs Health 1997;20:377-87.

33. O'Connor A, Edwards AG. The role of decision aids in promoting evidence-based patient choice. In: Edwards AG, Elwyn G, eds. Evidence-based patient choice: inevitable or impossible? Oxford: Oxford University Press, 2001:220-43.

34. Bekker HL, Thornton JG, Airey CM, et al. Informed decision making: an annotated bibliography and systematic review. Health Technol Assess 1999;3:1-156.
35. Mahmoodi N, Bekker HL, King N, et al. Decision aids' efficacy to support women's fertility preservation choices before cancer treatment: an environmental scan. European Society of Medical Decision Making Society Bi-Annual Conference. London: UK. June 2016.

36. Peate M, Meiser B, Friedlander M, et al. Development and pilot testing of a fertility decision aid for young women diagnosed with early breast cancer. Breast $J$ 2011;17:112-14.

37. Garvelink MM, ter Kuile MM, Fischer MJ, et al. Development of a decision aid about fertility preservation for women with breast cance in The Netherlands. J Psychosom Obstet Gynecol 2013;34:170-8.

38. Coulter A, Stilwell D, Kryworuchko J, et al. A systematic development process for patient decision aids. BMC Med Inform Decis Mak 2013;13(Suppl 2):S2.

39. Winterbottom AE, Gavaruzzi T, Bekker HL. Patient acceptability of the Yorkshire Dialysis Decision Aid (YoDDA) Booklet: a prospective non-randomised comparison study across 6 predialysis services. Pert Dial Int 2016;36:374-81.

40. Craig P, Dieppe P, Macintyre S, et al. Developing and evaluating complex interventions: the new Medical Research Council guidance. BMJ 2008;337:a1655

41. Purva Abhyankar P, Volk RJ, Blumenthal-Barby J, et al. Balancing the presentation of information and options in patient decision aids: an updated review. BMC Med Inform Decis Mak 2013;13(Suppl 2):S6

42. Stalmeier $\mathrm{P}$, Volk RJ, Abhyankar $\mathrm{P}$, et al. Balancing the presentation of information and options. In: Volk R, Llewellyn-Thomas $\mathrm{H}$. eds. Update of the International Patient Decision Aids Standards (IPDAS) Collaboration's background document. Chapter I, 2012. http://ipdas. ohri.ca/resources.html (accessed 20 Mar 2016)

43. Trevena LJ, Davey HM, Barratt A, et al. A systematic review on communicating with patients about evidence. J Evaluation Clin Pract 2006;12:13-23.

44. Trevena LJ, Zikmund-Fisher BJ, Edwards A, et al. Presenting quantitative information about decision outcomes: a risk communication primer for patient decision aid developers. BMC Med Inform Decis Mak 2013;13(Suppl 2):S7.

45. Barratt A, Trevena L, Davey HM, et al. Use of decision aids to support informed choices about screening. BMJ 2004;329:507-10.

46. O'Connor AM, Stacey D, Tugwell P, et al. Incorporating patient values. In: DiCenso A, Guyatt G, Ciliska D, eds. Evidence-based nursing: a guide to clinical practice. Toronto: Mosby, 2005;490-507.

47. Fagerlin A, Pignone M, Abhyankar $P$, et al. Clarifying values: an updated review. BMC Med Inform Decis Mak 2013;13(Suppl 2):S8.

48. Ley P, Florio T. The use of readability formulas in health care. Psychol Health Med 1996;1:7-28.

49. Leventhal $H$, Diefenbach $M$, Leventhal EA. Illness cognition: using common sense to understand treatment adherence and affect cognition interactions. Cognit Ther Res 1992;16:143-63.

50. Leventhal H, Benyamini $\mathrm{Y}$, Bownlee $\mathrm{S}$, et al. Illness Representations: Theoretical Foundations. In: Petrie KJ, Weinman JA, eds. Perceptions of health and illness: current research and applications. The Netherlands: Harwood Academic Publisher, 1997;19-46.

51. Doak LG, Doak CC, Meade CD. Strategies to improve cancer education materials. Oncol Nurse Forum 1996;23:1305-12.

52. Knapp CA, Quinn GP, Murphy DM. Assessing the reproductive concerns of children and adolescents with cancer: challenges and potential solutions. J Adolesc Young Adult Oncol 2011;1:31-5.

53. Quinn P, Vadaparampil ST, Sehovic I, et al. Patient and Family Tools to Aid in Education and decision Making About Oncofertility. In: Woodruff TK, Clayman ML, Waimey KE, eds. Oncofertility communication: sharing information and building relationships across disciplines. Springer: New York, 2014;35-47.

54. Jones G, Kennedy S, Barnard A, et al. Development of an endometriosis quality-of-life instrument: the endometriosis health profile-30. Obstet Gynecol 2001;98:258-64.

55. The British Psychological Society. Professional Practice Guidelines 1995. Report. Leicester, UK, 2001. https://www.bps.org.uk (accessed 22 Jun 2016).

56. Bennett C, Graham ID, Kristjansson E, et al. Validation of a preparation for decision making scale. Patient Educ Couns 2010;78:130-3.

57. Moores KL, Jones GL, Radley SC. Development of an instrument to measure face validity, feasibility and utility of patient questionnaire use during health care: the QQ-10. Int J Qual Health Care 2012;24:517-24.

58. Braun V, Clarke V. Using thematic analysis in psychology. Qual Res in Psychol 2006;3:77-101.

59. Elwyn G, Scholl I, Tietbohl C, et al. 'Many miles to go...': a systematic review of the implementation of patient decision support 
interventions into routine clinical practice. BMC Med Inform Decis Mak 2013;13(Suppl 2):S14.

60. Ottawa Hospital Research Institute. Patient Decision Aids Research Group. 2014. https://decisionaid.ohri.ca/cochinvent.php (accessed 27 Jan 2016).

61. Marteau TM, Bekker HL. The development of a six-item short-form of the state scale of the Spielberger State-Trait Anxiety Inventory (STAl). Br J Clin Psychol 1992;31(Pt 3):301-6.

62. The Euroqol Group. Euroqol- a new facility for the measurement of health-related quality-of-life. Health Policy 1990;16:199-208.

63. O'Connor AM. Stage of decision making. Ottawa: Ottawa Hospital Research Institute. 2000. https://decisionaid.ohri.ca/eval_stage.html (Accessed 27 Jan 2016).

64. O'Connor AM. Validation of a decisional conflict scale. Med Decis Making 1995;15:25-30.
65. Chamberlain K. Using grounded theory in health psychology. In: Murray M, Chamberlain K, eds. Qualitative health psychology. London: Sage, 1999:183-201.

66. Miles MB, Huberman AM. Qualitative data analysis: an expanded sourcebook. Los Angeles: Sage, 1994.

67. Brehaut JC, O'Connor AM, Wood TJ, et al. Validation of a decision regret scale. Med Decis Making 2003;23:281-92.

68. Ritchie J, Spencer L, O'Connor W. Carrying out qualitative analysis. In: Ritchie J, Lewis J, eds. Qualitative research practice: a guide for social science students and researchers. London: SAGE Publications Ltd, 2003: 220-62.

69. Elwyn G, Kreuwel I, Durand MA, et al. How to develop web-based decision support interventions for patients: a process map. Patient Educ Couns 2011;82:260-5. 